This article is now published in Cerebral Cortex:

Lynn, A., Wilkey, E. D., \& Price, G. R. (2021). Predicting Children's Math Skills from TaskBased and Resting-State Functional Brain Connectivity. Cerebral Cortex, 1-11, epub ahead of print. https://doi.org/https://doi.org/10.1093/cercor/bhab476 


\title{
Predicting children's math skills from task-based and resting-state functional brain connectivity
}

\author{
Andrew Lynn ${ }^{1}$, Eric D. Wilkey ${ }^{2}, \&$ Gavin R. Price ${ }^{1,3}$ \\ ${ }^{1}$ Department of Psychology and Human Development, Peabody College, Vanderbilt University, \\ Nashville, TN, USA \\ ${ }^{2}$ Brain \& Mind Institute, Western University, London, ON, Canada \\ ${ }^{3}$ Vanderbilt Brain Institute, Vanderbilt University, Nashville, TN, USA
}

\section{Acknowledgements}

We thank members of the Numerical Brain Lab at Vanderbilt University for recruitment and testing. We also thank Benjamin Conrad and Darren Yeo for feedback on this project. Finally, we thank the children and families that trusted us and made this research possible. Research was supported by NSF 1660816 (GP) and NSF 1750213 (GP). We have no known conflicts of interest to disclose.

Correspondence concerning this article should be addressed to Andrew Lynn, Department Psychology and Human Development, Vanderbilt University, 230 Appleton Place, Nashville, TN 37203. Contact Andrew.Lynn.1@vanderbilt.edu, 615-241-0894.

Data Availability: Processed data are available at https://osf.io/b4uej/.

\footnotetext{
Abstract

A critical goal of cognitive neuroscience is to predict behavior from neural structure and function, thereby providing crucial insight into who might benefit from clinical and/or educational
} 
interventions. Across development, the strength of functional connectivity among a distributed set of brain regions is associated with children's math skills. Therefore, in the present study we use Connectome-based Predictive Modeling to investigate whether functional connectivity during numerical processing and at rest predicts children's math skills $\left(N=31, M_{\text {age }}=9.21\right.$ years, 14 Female). Overall, we found that functional connectivity during symbolic number comparison and rest, but not during non-symbolic number comparison, predicts children's math skills. Each task revealed a largely distinct set of predictive connections distributed across canonical brain networks and major brain lobes. Most of these predictive connections were negatively correlated with children's math skills, such that weaker connectivity predicted better math skills. Notably, these predictive connections were largely non-overlapping across task states, suggesting children's math abilities may depend on state-dependent patterns of network segregation and/or regional specialization. Furthermore, the current predictive modeling approach moves beyond brainbehavior correlations and toward building models of brain connectivity that may eventually aid in predicting future math skills.

Keywords: childhood, development, math, functional connectivity, predictive modeling 
A critical goal of cognitive neuroscience is to predict behavior from neural structure and function. One promising approach is "Connectome-based predictive modeling" (CPM), in which an individual's behavior is predicted from their brain connectivity patterns (e.g., Shen et al., 2017). CPMs derived from functional brain connectivity during attention tasks have proven useful in predicting attention deficit hyperactivity disorder severity in childhood (Rosenberg et al., 2015). However, such predictive methods have not yet been applied in the domain of academic development. Early prediction of risk of low math achievement is crucial providing insight into which children might benefit from clinical and/or educational interventions. Therefore, in the present study we use CPM to investigate whether functional connectivity during numerical processing and at rest predicts children's math skills.

Predictive modeling leverages the relation between two variables (e.g., brain connectivity and behavior) in a dataset to make a prediction about novel data (Gabrieli et al., 2015; Rosenberg et al., 2018). In the present study, we refer to "prediction" as the output of a model when applied to a novel datapoint and "correlation" as the statistical measure of association between two variables across a set of data. A key goal of developmental and educational neuroscience is to predict a future behavior from earlier brain and behavioral measures. However, predictive modeling may also be used to examine individual differences at a given time in development. We have focused on predicting $3^{\text {rd }}$ and $4^{\text {th }}$ grader's (8-10-year-olds) math skills from their concurrent brain connectivity because this is a time in academic development when individual differences in math skills begin to emerge (Geary \& Hoard, 2005; Jordan \& Hanich, 2003).

The extant functional connectivity literature shows that individual's math skills are correlated with functional connectivity strength between a distributed set of frontal, parietal, and temporooccipital brain regions. Key math-related brain regions include the inferior frontal gyrus 
(IFG), intraparietal sulcus (IPS), and inferior temporal number area (ITNA) located in the inferior temporal gyrus (ITG) (Arsalidou \& Taylor, 2011; D. J. Yeo et al., 2017). Subdivisions of these regions are distributed across several canonical networks, including the Frontoparietal, Dorsal Attention and Ventral Attention networks (B. T. Yeo et al., 2011), suggesting several brain networks may be involved in the development of math skills. Across childhood, stronger frontoparietal connectivity during symbolic number processing is associated with better math skills (Park et al., 2014). Some studies show that during calculation, stronger intraparietal functional connectivity is related to better math skills (Battista et al., 2018; Park et al., 2013), but others show that weaker intraparietal and frontoparietal connectivity is associated with better math skills (Rosenberg-Lee et al., 2015). Similarly, weaker temporooccipital functional connectivity during number processing is related to better math skills in adulthood (Bulthé et al., 2019). Across childhood, stronger resting-state connectivity within frontal and between frontal and parietal regions is correlated with better counting, numerical processing, and calculation skills (Zhang et al., 2019). Moreover, stronger intraparietal and weaker frontoparietal resting-state connectivity in $1^{\text {st }}$ grade is associated with children's calculation skills in $2^{\text {nd }}$ grade (Price et al., 2018). Across childhood, stronger resting-state connectivity between temporooccipital and frontal, and parietal regions is associated with better calculation skills (Evans et al., 2015; Nemmi et al., 2018). The extant literature, therefore, presents an inconsistent web of findings showing both stronger and weaker functional connectivity supports better math skills in childhood.

One possible explanation for this inconsistency is that the relation between functional connectivity and children's math skills may differ depending on the functional connectivity taskstate (Finn et al., 2017; Geerligs et al., 2015). Both behavioral and brain data suggest that nonsymbolic and symbolic magnitude comparison abilities support math skills across development 
(e.g., De Smedt et al., 2013), with non-symbolic magnitude processing setting the foundation for symbolic magnitude processing abilities across childhood (Price \& Fuchs, 2016). It is, as yet, unclear whether patterns of functional connectivity differentially predict children's math skills during non-symbolic and symbolic number comparison tasks and at rest. If the same connectivity profile predicts math skills across tasks and rest, it would suggest that a stable pattern of functional connectivity, or trait-level neural architecture, may provide the foundation for each child's math skills. On the other hand, if varying patterns of functional connectivity across these task and rest states predict math skills, it may reflect a flexibility in network organization that supports the acquisition of math skills.

Here, we used CPM (Rosenberg et al., 2015; Shen et al., 2017) to test whether functional connectivity during symbolic and non-symbolic number comparison, and during rest, predicts children's math skills measures outside the scanner. CPM leverages leave-one-out cross validation (LOOCV) to: 1) select all functional connections correlated with a behavior of interest (i.e., math skills), 2) train a linear model to fit the sum of connection strengths and behavioral variable, and 3) predict the behavior of a held-out participant from their functional connectivity values using the trained linear model. In using CPM, the present study moves beyond brain-behavior correlation to predict children's math skills from their functional connectivity during different task-states and at rest. 


\section{Materials and Methods}

\section{Participants}

The final sample included 31 typically achieving 8- to 10-year-old children (14 Female). Children completed a behavioral visit and a neuroimaging visit. An additional 5 children that completed the neuroimaging visit were removed from analyses due to excessive motion during resting-state. We defined excessive motion as at least half of the scan volumes (i.e., TRs) with DVARS $>3 \mathrm{deg}$ and/or FD $>0.5 \mathrm{~mm}$. The current sample partially overlaps with a previously published sample (Wilkey \& Price, 2019), from which we include only children whom completed both symbolic and non-symbolic number comparison task scans and resting state scans. Children's parents provided consent and children provided their assent in accordance with our University IRB policies. Prior to neuroimaging, children were acclimated to mock MRI scanner.

\section{Experimental Design and Statistical Analyses}

\section{Behavioral Visit}

Children first completed a behavioral visit in which they completed several tasks, including the Woodcock-Johnson III Tests of Achievement (WJ-III) (Woodcock et al., 2001) and the Kaufman Brief Intelligence Test, second edition (KBIT) (Kaufman \& Kaufman, 2004). We derived separate composite math and reading scores from the WJ-III and full-scale IQ scores from the KBIT.

Math Skills. We measured children's math skills using the WJ-III Applied Problems, Calculation and Math Fluency subtests. The Applied Problems subtest measures children's ability to analyze and solve math problems in an untimed task where they hear a math word problem and must correctly select the relevant numerical information and mathematical operation to find the answer. The Calculation subtest measures children's ability to perform math computations in an 
untimed task where they complete as many calculation problems as possible, ranging from simple arithmetic to calculus and increased in difficulty with each consecutive problem. The Math Fluency subtest measures children's ability to quickly solve calculation problems in a 3-minute timed task where they complete as many simple addition, subtraction, and multiplication problems as possible. Subtest scores were first normed to the child's age and then averaged to create composite math score for each child.

Reading Skills. We measured children's reading skills using the WJ-III Letter-Word Identification and Passage Comprehension subtests. The Letter-Word Identification subtest measures children/s ability to first identify target letters and words among distracting letters, words, and pictures, and then to read words of increasing difficulty. The Passage Comprehension subtest measures children's ability to first match pictures of common objects to symbols, then to read words or phrases and choose the matching picture, and finally to fill in missing words in sentences and paragraphs of increasing difficulty. Subtest scores were first normed to the child's age and then averaged to create composite reading score for each child.

Intelligence Quotient (IQ). We calculated Full scale IQ estimates for each child based on the KBIT. Full-scale IQ is a composite score of verbal IQ and non-verbal IQ. Verbal IQ is measured using picture-based vocabulary and riddles, and non-verbal IQ is measured using matrix reasoning questions.

\section{Neuroimaging Visit}

Symbolic and Non-symbolic Number Comparison Tasks. We instructed children to lay motionless while they completed a symbolic and non-symbolic number comparison task during fMRI scanning. We counterbalanced symbolic and non-symbolic number comparison tasks across 
all participants. We asked participants to indicate which of two simultaneously presented Arabic digits (symbolic) or sets of dots (non-symbolic) was larger in magnitude by responding with a button press on either a left-hand or right-hand button box (i.e. two button boxes were used). We presented target stimuli for $1250 \mathrm{~ms}$ followed by a fixation line for $3250 \mathrm{~ms}$ to $6250 \mathrm{~ms}(1000 \mathrm{~ms}$ steps, $M=4750 \mathrm{~ms}$ ). We recorded RTs and error rates (ER) to calculate a 'Performance' metric, $P$ $=\mathrm{RT}(1+2 \mathrm{ER})$, which corrects children's RTs by their ER and controls for speed/accuracy tradeoffs (Lyons et al., 2014). Thus, a smaller P metric corresponds to better performance. Note that accuracy on both the symbolic $(\mathrm{M}=91.7 \%, \mathrm{SD}=5.8 \%)$ and non-symbolic tasks $(\mathrm{M}=86.5 \%$, SD $=9.9 \%$ ) was well above chance.

The numerosity ratio (smaller number divided by larger number) between the two stimuli varied from smaller and easier (0.286 to 0.375$)$ to larger and harder (0.625 to 0.714$)$. Arabic digits ranged from 1 to 9 and the number of dots in each set ranged from 5 to 21 . Each task included a total of 80 trials (40 easy ratio, 40 hard ratio). On half of the trials the physical size of the stimulus was congruent with the numerosity (large 7, small 3) and on the other half the size of the stimulus was incongruent (small 7, large 3). For the non-symbolic number comparison task stimulus "size" was measured by convex hull, total surface area and dot diameter (Gebuis \& Reynvoet, 2011).

Resting State. Children completed resting state scans after having completed both number comparison tasks. Children were instructed to lay motionless with their eyes open while a fixation cross was presented on the screen.

Image Acquisition, Preprocessing, and Nuisance Regression. We acquired all MR imaging with a Phillips Achieva 3T MR scanner using a 32-channel head coil. Children watched a cartoon movie while we acquired high-resolution anatomical images using a T1-weighted Magnetization Prepared Rapid Gradient Recalled Echo sequence according to the following 
specifications: $\mathrm{TR}=8.929 \mathrm{~s} ; \mathrm{TE}=4.61 \mathrm{~ms}$; flip angle $=8^{\circ} ; 170$ sagittal slices with no inter-slice gap; voxel size $=1 \times 1 \times 1 \mathrm{~mm}$; imaging matrix $=256 \times 256$; acquisition time $=264.8 \mathrm{~s}$. We oriented scans in the anterior-posterior commissure plane.

We acquired whole-brain functional images using a multislice 2D SENSE T2* gradientecho, echo planar imaging pulse sequence in the axial plane with the following parameters: Slices $=40$; Repetition Time $(\mathrm{TR})=2000 \mathrm{~ms}$; Echo Time $(\mathrm{TE})=25 \mathrm{~ms}$; voxel size $=2.5 \times 2.5 \times 3 \mathrm{~mm}$ with an inter-slice gap of $0.25 \mathrm{~mm}$; field of view $=240 \times 129.75 \times 240 \mathrm{~mm}$; imaging matrix $=96$ $\times$ 96; flip angle $=90^{\circ}$; SENSE factor $=2.5$. Symbolic and non-symbolic task scans were approximately 9 minutes in total (4.5 minutes per run), and the resting scan was approximately 7.5 minutes.

We preprocessed images using FreeSurfer v7.1.1 (Ségonne et al., 2007) and the CONN Toolbox v20 (Whitfield-Gabrieli \& Nieto-Castanon, 2012) for SPM in MATLAB R2019b. We first segmented anatomical images using the FreeSurfer cortical reconstruction process (i.e., reconall). We visually inspected and manually edited grey matter and white matter segmentations for common errors (e.g., skull strip errors). We then preprocessed functional imaging data using the CONN toolbox preprocessing pipeline for surface-based analyses. First, we aligned functional volumes and subsequently corrected for slice-timing. Then, we co-registered each child's functional images to their anatomical image using non-linear registration and then resampled to children's FreeSurfer structural cortical surface. Finally, we smoothed surface data using iterative spatial diffusion smoothing with 40 iterations to approximate 8mm FWHM kernel (Hagler et al., 2006).

Following preprocessing, we employed nuisance regression to correct for in-scanner motion and remove task activation effects using CONN Toolbox for SPM. We included the 
following parameters: 1) first 5 components and derivatives of both CSF and WM signals, 2) 24 movement parameters derived from children's realignment (i.e., x, y, z, roll, pitch, yaw, derivatives, quadratic expansion), 3) global signal, and 4) framewise displacement (FD). We also included task event onsets convolved with a canonical hemodynamic response function (HRF) to remove the effect of task activation. We then band-pass filtered $(0.008<\mathrm{f}<0.09 \mathrm{~Hz})$ functional surface data.

We chose short runs for each task so children could take breaks and therefore minimize the in-scanner motion (e.g., Meissner et al., 2020). Because we had short runs, we also aimed to keep as much data as possible. Spike regression/scrubbing reduces temporal degrees of freedom and may bias functional connectivity estimates across the sample (Ciric et al., 2017). Therefore, we explored the impact of two temporal censoring approaches separately: "spike regression" (DVARS $>3 \mathrm{deg}$ and/or FD $>0.5 \mathrm{~mm}$ ) and "despiking" (prior to regression). There are several ways to define a successful denoising approach (see Ciric et al., 2017; Satterthwaite et al., 2019). One way is to determine which approach resulted in minimizing relation between CONN Toolbox quality control (QC) measures (i.e., mean/max motion, mean/max global signal change, number of TRs without motion, and mean framewise displacement) and functional connectivity (FC) values. Stronger QC-FC correlations at the group levels would indicate that a denoising approach did not adequately remove motion-related noise, but weaker QC-FC correlations would indicate the approach adequately removed motion-related noise. Thus, a successful denoising approach would result in a distribution of QC-FC correlations centered around zero. A secondary benchmark was that individual's connectivity values were normally distributed around zero. Overall, visual inspection QC-FC correlation distributions and individual connectivity value distributions indicated that timeseries despiking, rather than spike regression, resulted in a better denoising (see 
Supplemental Figure 1). Therefore, our complete motion correction pipeline included timeseries despiking followed by nuisance regression as outlined above.

Following preprocessing and nuisance regression, we utilized the CONN Toolbox v20 to extract the raw signal timeseries from each of the 210 cortical ROIs from the connectivity-derived and biologically plausible Brainnetome Atlas (Fan et al., 2016). For each child, we then calculated the Fisher z-transformed correlation coefficient between the timeseries of all regions, which resulted in a 210 by 210 functional connectivity matrix. These child-level $z$-score connectivity matrices were then submitted to subsequent analyses. See Supplementary Figure 2 for surfacebased Brainnetome atlas with Yeo et al., (2011) network labels.

Connectome-based Predictive Modeling (CPM). We used separate CPMs to predict children's composite math skills (measured outside the scanner) from connectivity during symbolic number comparison, non-symbolic number comparison, and resting state (eyes open). CPM employs leave-one-out cross-validation (LOOCV) to predict a held-out (novel) child's behavior (e.g., math skills) from an independent set of connectivity values across three main steps: 1) feature selection, 2) model fitting, and 3) behavior prediction (Rosenberg et al., 2015; Shen et al., 2017). During feature selection, we selected connections correlated with composite math skills scores in the training set $(p<.01)$, controlling for children's age and mean FD. Note that during the feature selection step, connection strength may be positively or negatively correlated with the behavior of interest. During the model fitting step, we therefore trained a general linear model (GLM) with one parameter for the sum of positive features and one for the sum of negative features. During behavior prediction, we used the fitted GLM to predict the held-out child's composite math skills score. We repeated this process until every child was held out, yielding a predicted composite math score for each child. 
To assess each model, we calculated the Pearson correlation between predicted and observed composite math skills scores and followed up with permutation testing (iterations $=$ 10,000). We report both standard $p$-values associated with the correlation distribution (corrected for multiple comparisons) and permutation test $p$-values. To calculate permutation $p$-values, we randomly permuted children's composite math scores and ran the same CPM procedure 10,000 times to create a null distribution of $r$ values. Then we divided the number of times the null $r$ values were greater than or equal to the observed $r$ value by the total number of permutations. 


\section{Results}

\section{Correlations Among Behavioral Measures}

We computed Pearson correlations to determine the relation between age (years), composite math score, composite reading score, full scale IQ, and motion for each task (mean FD). See Table 1 for all statistics. Better math skills were associated with better reading skills and higher full-scale IQ. Better reading skills were also associated with higher full-scale IQ and less inscanner motion across all three tasks. In-scanner motion among the three tasks was positively correlated.

\section{[Table 1 here]}

\section{Connectome-based Predictive Modeling}

We used CPM to determine whether functional connectivity during each task separately (i.e., symbolic, non-symbolic, and rest) predicts children's composite math skills (e.g., WJ-III). To account for the influence of age-related changes and in-scanner motion on functional connectivity, we include age (years) and mean FD as covariates during feature selection. It is important to highlight that, because each child serves as the held-out participant across different LOOCV rounds, the exact connections that predict each children's composite math skills likely vary. In other words, the set of connections whose strength is summed to predict child A may only partially overlap with the set of connections whose strength is summed to predict child B. To characterize which functional connections consistently predicted children's composite math skills across the sample, we identified connections whose strength was selected by the model across all LOOCV rounds. This resulted in one set of "consistent connections" for each model. Note that these 
connections are a subset of those identified across each LOOCV round and are selected for illustrative purposes.

To observe whether connectivity predicts composite math skills similarly or differently across each task state, we identify which consistent connections predict composite math skills across more than one task-state. If similar connections predict math scores across multiple taskstate, then children's trait-dependent connectivity may underlie individual differences in children's composite math skills. However, if different connections predict composite math skills for each task-state, then state-dependent connectivity may underlie these individual differences.

[Figure 1 here]

\section{Functional connectivity during symbolic number comparison predicts children's math skills}

CPM revealed 14 positive and 59 negative consistent connections during symbolic number comparison that together significantly predicted children's composite math score (Figure $1 ; r=$ .535 , Bonferroni-corrected $p=.006$, permutation $p=.01)$. The most common connections whose strength was positively correlated with children's math skills were between the Visual and Default Mode networks (29\%). The regions with the largest number of positive connections were the $\mathrm{R}$ posterior Superior Temporal Sulcus $(n=3)$, R Precuneus $(n=3)$, and L Precuneus $(n=3)$. See Figure 2 for depictions of consistently predictive connections and their distribution across networks.

The most common connections whose strength was negatively correlated with children's math skills were also between the Visual and Default Mode networks (19\%) and between the Dorsal Attention and Default Mode networks (10\%). The regions with the largest number of 
negatively correlated connections were the L Middle Frontal Gyrus (MFG, n = 9), Left Superior Frontal Gyrus (SFG, $n=9)$, R SFG $(n=4)$, R Lateral Occipital Gyrus (LOC, $n=8)$, L Precentral Gyrus (PrG, n=6), R Middle Temporal Gyrus (MTG, n=6), L Insula ( $\mathrm{n}=5$ ), L Parahippocampal Gyrus (PhG, $n=5)$, R Inferior Parietal Lobule (IPL, n=5), L IPL ( $\mathrm{n}=4$ ), L Inferior Frontal Gyrus (IFG, $n=4)$, R Superior Parietal Lobule (SPL, $n=4)$, R Cingulate Gyrus (CG, $n=4)$, and the L CG $(n=4)$. See Supplementary Table 1 extended data for detailed list of consistently predictive connections.

Using the same approach, we found that functional connectivity during symbolic number comparison did not predict children's composite reading skills $(r=.245, p=.183$, permutation $p$ $=.156)$ or their full-scale IQ $(r=.262, p=.154$, permutation $p=.138)$.

[Figure 2 here]

Functional connectivity during non-symbolic number comparison does not predict children's math skills

During non-symbolic number comparison, functional connectivity did not predict children' composite math score outside the scanner (Figure $1 ; r=.154, p=.407$, permutation test $p=.237$ ). We also found that functional connectivity during non-symbolic number comparison did not predict children's composite reading skills $(r=.230, p=.213$, permutation $p=.152)$ or their fullscale IQ $(r=-.094, p=.616$, permutation $p=.561)$. Thus, we found no evidence that whole-brain functional connectivity during non-symbolic number comparison predicts children's math skills, reading skills or IQ. 
CPM revealed 6 positive and 38 negative consistent connections during resting state that together significantly predicted children's composite math scores (Figure $1 ; r=.541$, Bonferronicorrected $p=.005$, permutation test $p=.008$ ). The most common connections whose strength was positively correlated with children's math skills were between the Visual and Frontoparietal networks (33\%). The region with the largest number of positively correlated connections was the R Middle Frontal Gyrus $(n=3)$. Figure 3 for depictions of consistently predictive connections and their distribution across networks.

The most common connections whose strength was negatively correlated with children's math skills were within the Frontoparietal network (13\%) and between the Frontoparietal and Dorsal Attention networks (13\%). The regions with the largest number of negatively correlated connections were the R IFG $(n=10), \operatorname{L~IFG~}(n=6), \operatorname{L~MFG~}(n=6), \operatorname{R}$ MFG $(n=4), \operatorname{IPL}(n=$ 5), R Insula ( $\mathrm{n}=5)$, L Inferior Temporal Gyrus (ITG, $n=5)$, R ITG ( $\mathrm{n}=4), \mathrm{R}$ PrG $(\mathrm{n}=5)$, and L $\operatorname{PrG}(n=4)$. See Supplementary Table 2 extended data for detailed list of consistently predictive connections.

Using the same approach, we found that functional connectivity during rest did not predict children's composite reading skills $(r=.191, p=.303$, permutation $p=.207)$ or their full-scale IQ $(r=-.238, p=.198$, permutation $p=.757)$

[Figure 3 here]

\section{Predictive connections are distinct across different tasks}

So far, we have demonstrated that distributed sets of functional connections during symbolic number comparison and rest predict children's composite math scores. However, it is unclear whether the same or different connections are predictive of math skills across these task 
states. Therefore, we identified consistent connections that negatively predicted children's math skills during more than one task and/or at rest.

We found only one connection, between the $\mathrm{R}$ cingulate gyrus and $\mathrm{R}$ insula, negatively correlated with children's math skills across both symbolic number comparison and rest. We found no positively correlated connections shared between symbolic and rest. Additionally, we found no connections whose relationship with children's math skills switched direction from one task to another (e.g., positively correlated during symbolic number comparison, but negatively correlated during rest). The general lack of predictive connectivity overlap between tasks suggests math skills may be related to distinct patterns of cortical connectivity across different tasks.

\section{Summary}

Overall, we found that functional connectivity during symbolic number comparison and rest, but not during non-symbolic number comparison, predicts children's math skills. Within the same task state, we observed connections whose strength was positively correlated with children's math skills and those whose strength was negatively correlated with children's math skills. In both symbolic number comparison and resting state, we found more connections whose strength was negatively correlated with children's math skills than whose strength was positively correlated. During symbolic number comparison we found that many of the negatively correlated connections were between the Default Mode and Visual networks and between the Default Mode and Dorsal Attention networks. During Rest were found that many of the negatively correlated connections were within the Frontoparietal network and between the Frontoparietal and Dorsal Attention networks. Notably, these predictive connections were largely non-overlapping across task states. 


\section{Discussion}

Here, we used CPM to show that functional connectivity during both symbolic number comparison and resting state predicts children's math skills. Each task revealed a largely distinct set of predictive connections distributed across canonical brain networks and major brain lobes. Most predictive connections were negatively correlated with children's math skills, such that weaker connectivity predicted better math skills. Through the lens of canonical brain networks, most connections implicated the Default Mode, Dorsal Attention, and Visual networks during symbolic number processing, and the Frontoparietal and Dorsal Attention networks during resting state. Through the lens of individual brain regions, many predictive connections implicated key regions typically associated with higher-order cognition and numerical processing, including, among others, the IFG, MFG, IPL, and ITG.

This is first study to move beyond merely correlational measures of brain-behavior relationships and predict children's math skills from functional connectivity. The extant mathrelated functional connectivity literature is explanatory rather than predictive. We are in no way suggesting that these approaches are not valuable. Descriptive and explanatory approaches have taught us much about the brain, development, and cognition, and continue to be valuable for answering certain types of questions. However, predicting individual differences in math skills is important to better inform educators and clinicians about who may need intervention to remediate math skills across development. While our results suggest that a few minutes of resting-state fMRI data may be sufficient for predicting children's concurrent math skills, this is simply the foundation for constructing a generalizable model of functional connectivity that may eventually aide in predicting children's future math skills. It is important to note that in the current study children were in $3^{\text {rd }}$ and $4^{\text {th }}$ grade, a time when robust math skills differences begin to emerge (Geary $\&$ 
Hoard, 2005; Jordan \& Hanich, 2003). Thus, it remains unclear whether this model will generalize to earlier points in development when there is less variability in individual differences in math skills. Future longitudinal should examine whether and when functional connectivity first becomes predictive of children's later math skills, which may in turn inform the timing of intervention for math disabilities.

Most work examining math-related functional connectivity identifies a few regions of interest (ROIs) either functionally (e.g., activation across relevant task conditions) or anatomically (e.g., cytoarchitectonically) then correlates activity patterns in each ROI with the rest of the brain (Battista et al., 2018; Chang et al., 2016; Jolles et al., 2016; Nemmi et al., 2018; Park et al., 2014; Price et al., 2018). This approach is valuable for initially identifying brain networks and circuits underlying math abilities. However, it also limits the scope of the functional connectivity space to only those connections with an ROIs and makes it challenging to integrate findings because ROIs and the methods for defining them typically differ across studies. A few studies examine mathrelated connectivity among a circumscribed set of regions (Emerson \& Cantlon, 2012; RosenbergLee et al., 2011; Zhang et al., 2019), but these regions are also inconsistent across studies. The current approach, which defines brain regions based on patterns of anatomical and functional connectivity (i.e., Brainnetome atlas) (Fan et al., 2016), effectively expands on the multiple ROI approach to consider connections between all brain regions as potential predictors of children's math skills, allowing for the possibility of revealing connections related to math skills that were not previously considered or tested. To the extent that future studies adopt the current approach, examining functional connectivity between an agreed upon set of brain regions will facilitate integrating findings across the literature to identify points of reproducibility and novelty. Note, however, that it is possible that our chosen brain atlas may mask additional predictive power 
available from other connectivity configurations. Future work should compare several brain atlases to identify those that uncover the most predictive power, especially those that consider structural and functional differences across development.

To that end, our findings are broadly consistent with previous research showing that weaker frontal and parietal functional connectivity is correlated with better math skills (Jolles et al., 2016; Price et al., 2018; Rosenberg-Lee et al., 2015; Zhao et al., 2019). Across symbolic number comparison and resting state, we found that weaker connectivity within and between key regions associated with numerical cognition include the IFG, SPL, IPL, and ITG (Arsalidou \& Taylor, 2011; D. J. Yeo et al., 2017) is predictive of better math skills during symbolic number comparison and at rest. Within our canonical network definitions, subdivisions of these regions were distributed across several networks, including the Frontoparietal, Dorsal Attention, Ventral Attention, and Default Mode networks. During rest, many of the negatively predictive connections were between the IFG, MFG, IPL, and ITG, which connected regions within the Frontoparietal network and between the Frontoparietal and Dorsal Attention networks. However, our whole-brain ROI approach also revealed that, during symbolic number comparison, many of the negatively predictive connections occurred between the MFG and occipital cortex, which connected the Default Mode and Visual networks.

The current approach is limited in its ability to provide insight into the neural mechanisms underlying math skills. However, a benefit of the current approach is that we have identified sets of connections whose strength is either positively or negatively predictive of children's math skills, which allow us to make mechanistic predictions that can be tested in future work. Stronger connectivity between visual regions and those spanning the frontal and parietal lobes may reflect network integration and stronger attentional regulation of sensory processing regions. Weaker 
connectivity between key math-related and domain-general regions may reflect network segregation, regional specialization, and/or a reduction in the required level of cognitive engagement for higher math achievers. Moreover, that we found largely distinct sets of these connections across several task states that are thought to support math skills suggests that that brain states underlying basic numerical processing may aid in children's higher order math skills. Thus, the current study points to task-dependent network integration and segregation among math-related regions and domain-general regions (e.g., visual cortex), as possible neural mechanisms supporting children's math skills. Of course, future experimental work is needed to confirm these predictions.

The current study is the first to examine the relation between math skills and both taskbased and resting-state connectivity in the same cohort of children. While we found that the precise cortical connections that predicted math skills largely differed between symbolic number comparison and resting state, it remains unclear whether subcortical connections and subcorticalcortical connections predict math skills across different task states. Nevertheless, the utility of taskbased CPM remains crucial for insight to mechanistic questions. That is, children's math skills may depend on state-dependent, rather than trait-dependent, patterns of distributed whole-brain functional connectivity patterns. Moreover, this task-dependent variability may explain the disparate findings across the extant literature regarding which specific regional connections correlate with math skills. Whether and how changes in connectivity across task demands predict children's math skills remains an open question. Future work needs to consider changes in connectivity strength as input to the CPM model rather than connectivity strength per se to determine how reconfigurations in state-dependent functional network architecture predict children's math skills. 
The predictive capacity of functional connectivity during symbolic number comparison and at rest seems to be specific to math skills. That is, functional connectivity did not also predict children's reading skills or general cognitive ability. We were surprised to find that resting-state functional connectivity did not predict children's reading skills and general cognitive abilities. It is worth noting, however, that resting-state data were collected following two numerical comparison tasks and a flanker task. Children also previously visited the lab in which they completed a battery of math-related tasks. Therefore, we speculate that children's resting-state functional connectivity may contain residual patterns of connectivity related to numerical processing, decreasing the predictive power of these functional connections for predicting reading skill and general cognitive ability (e.g., Tung et al., 2013). Future work should explore the role of reading-related task states in predicting reading skills by collecting resting-state data directly following both reading- and math-related task and comparing the predictive capacity of these two resting-state scans.

The present study has several limitations that need to be addressed to further develop the current predictive model of brain connectivity for children's math skills. First, we have only provided evidence for internal validation. Future work will use the currently established model to predict children's math skills in a novel dataset to assess model generalizability and provide external validation (Gabrieli et al., 2015). The limited number of developmental numerical cognition studies and lack of large numerical cognition fMRI datasets limits out ability to conduct such external validation analyses. Second, our sample size is relatively small for modeling of individual differences; however, previous predictive models have been derived from small sample sizes and show promise in generalizing across samples (e.g., Rosenberg et al., 2015). Future work should ideally include larger samples $(\mathrm{N}>100)$ to increase power and allow for more complex 
models to be fit (Dubois \& Adolphs, 2016), although there are of course logistical obstacles to collecting such large samples. Future iterations of large-scale neuroimaging studies such as the Adolescent Brain and Cognitive Development (ABCD) and Human Connectome Project Development (HCP-D) studies should consider including measures of math and reading skills. Including measures of academic skills in these large studies will also work to address the first limitation because large samples may be split into test and replication samples to provide internal and external validity. Finally, our data are cross-sectional and can only speak to the predictive power of functional connectivity for children's concurrent math skills. If predictive models of brain connectivity are to provide value in diagnosing and identifying children in need of early intervention for dyscalculia and other math-related developmental disorders, future work must leverage longitudinal data to predict children's future math skills from earlier functional connectivity, ideally before robust individual differences emerge.

\section{Conclusions}

Our findings demonstrate that weaker functional connectivity distributed across the whole brain and weaker connectivity between key math-related brain regions and between domaingeneral grain regions predicts better math skills in childhood. Critically, predictive connections largely differed between tasks, suggesting children's math abilities may depend on state-dependent (rather than trait-dependent) patterns of functional connectivity that tap into different canonical networks according to the task-state. These findings provide a framework to compare findings across future studies and the foundation for constructing a generalizable model of functional connectivity that may eventually aid in predicting children's future math skills. 


\section{References}

Arsalidou, M., \& Taylor, M. J. (2011). Is $2+2=4$ ? Meta-analyses of brain areas needed for numbers and calculations. NeuroImage, 54(3), 2382-2393. https://doi.org/10.1016/j.neuroimage.2010.10.009

Battista, C., Evans, T. M., Ngoon, T. J., Chen, T., Chen, L., Kochalka, J., \& Menon, V. (2018). Mechanisms of interactive specialization and emergence of functional brain circuits supporting cognitive development in children. Npj Science of Learning, 3(1), 1. https://doi.org/10.1038/s41539-017-0017-2

Bulthé, J., Prinsen, J., Vanderauwera, J., Duyck, S., Daniels, N., Gillebert, C. R., Mantini, D., Op de Beeck, H. P., \& De Smedt, B. (2019). Multi-method brain imaging reveals impaired representations of number as well as altered connectivity in adults with dyscalculia. NeuroImage, 190(June 2018), 289-302. https://doi.org/10.1016/j.neuroimage.2018.06.012

Chang, T. T., Metcalfe, A. W. S., Padmanabhan, A., Chen, T., \& Menon, V. (2016). Heterogeneous and nonlinear development of human posterior parietal cortex function. NeuroImage, 126, 184-195. https://doi.org/10.1016/j.neuroimage.2015.11.053

Ciric, R., Wolf, D. H., Power, J. D., Roalf, D. R., Baum, G. L., Ruparel, K., Shinohara, R. T., Elliott, M. A., Eickhoff, S. B., Davatzikos, C., Gur, R. C., Gur, R. E., Bassett, D. S., \& Satterthwaite, T. D. (2017). Benchmarking of participant-level confound regression strategies for the control of motion artifact in studies of functional connectivity. NeuroImage, 154(March), 174-187. https://doi.org/10.1016/j.neuroimage.2017.03.020

De Smedt, B., Noël, M. P., Gilmore, C., \& Ansari, D. (2013). How do symbolic and nonsymbolic numerical magnitude processing skills relate to individual differences in children's mathematical skills? A review of evidence from brain and behavior. Trends in 
Neuroscience and Education, 2(2), 48-55. https://doi.org/10.1016/j.tine.2013.06.001

Dubois, J., \& Adolphs, R. (2016). Building a Science of Individual Differences from fMRI. Trends in Cognitive Sciences, 20(6), 425-443. https://doi.org/10.1016/j.tics.2016.03.014

Emerson, R. W., \& Cantlon, J. F. (2012). Early math achievement and functional connectivity in the fronto-parietal network. Developmental Cognitive Neuroscience, 2(SUPPL. 1), S139S151. https://doi.org/10.1016/j.den.2011.11.003

Evans, T. M., Kochalka, J., Ngoon, T. J., Wu, S. S., Qin, S., Battista, C., \& Menon, V. (2015). Brain structural integrity and intrinsic functional connectivity forecast 6 year longitudinal growth in children's numerical abilities. Journal of Neuroscience, 35(33), 11743-11750. https://doi.org/10.1523/JNEUROSCI.0216-15.2015

Fan, L., Li, H., Zhuo, J., Zhang, Y., Wang, J., Chen, L., Yang, Z., Chu, C., Xie, S., Laird, A. R., Fox, P. T., Eickhoff, S. B., Yu, C., \& Jiang, T. (2016). The Human Brainnetome Atlas: A New Brain Atlas Based on Connectional Architecture. Cerebral Cortex, 26(8), 3508-3526. https://doi.org/10.1093/cercor/bhw157

Finn, E. S., Scheinost, D., Finn, D. M., Shen, X., Papademetris, X., \& Constable, R. T. (2017). Can brain state be manipulated to emphasize individual differences in functional connectivity? NeuroImage, 160(March), 140-151. https://doi.org/10.1016/j.neuroimage.2017.03.064

Gabrieli, J. D. E., Ghosh, S. S., \& Whitfield-Gabrieli, S. (2015). Prediction as a humanitarian and pragmatic contribution from human cognitive neuroscience. Neuron, 85(1), 11-26. https://doi.org/10.1016/j.neuron.2014.10.047

Geary, D. C., \& Hoard, M. K. (2005). Learning disabilities in arithmetic and mathematics: Theoretical and empirical perspectives. In J. I. D. Campbell (Ed.), The Handbook of 
Mathematical Cognition (pp. 253-267). Psychology Press.

https://doi.org/10.4324/9780203998045-24

Gebuis, T., \& Reynvoet, B. (2011). Generating nonsymbolic number stimuli. Behavior Research Methods, 43(4), 981-986. https://doi.org/10.3758/s13428-011-0097-5

Geerligs, L., Rubinov, M., Tyler, L. K., Brayne, C., Bullmore, E. T., Calder, A. C., Cusack, R., Dalgleish, T., Duncan, J., Henson, R. N., Matthews, F. E., Marslen-Wilson, W. D., Rowe, J. B., Shafto, M. A., Campbell, K., Cheung, T., Davis, S., Geerligs, L., Kievit, R., ... Henson, R. N. (2015). State and trait components of functional connectivity: Individual differences vary with mental state. Journal of Neuroscience, 35(41), 13949-13961.

https://doi.org/10.1523/JNEUROSCI.1324-15.2015

Hagler, D. J., Saygin, A. P., \& Sereno, M. I. (2006). Smoothing and cluster thresholding for cortical surface-based group analysis of fMRI data. NeuroImage, 33(4), 1093-1103. https://doi.org/10.1016/j.neuroimage.2006.07.036

Jolles, D., Supekar, K., Richardson, J., Tenison, C., Ashkenazi, S., Rosenberg-Lee, M., Fuchs, L., \& Menon, V. (2016). Reconfiguration of parietal circuits with cognitive tutoring in elementary school children. Cortex, 83, 231-245.

https://doi.org/10.1016/j.cortex.2016.08.004

Jordan, N. C., \& Hanich, L. B. (2003). Characteristics of Children with Moderate Mathematics Deficiencies: A Longitudinal Perspective. Learning Disabilities Research and Practice, 18(4), 213-221. https://doi.org/10.1111/1540-5826.00076

Kaufman, A. S., \& Kaufman, N. L. (2004). Kaufman Brief Intelligence Test, second edition. American Guidance Service.

Lyons, I. M., Price, G. R., Vaessen, A., Blomert, L., \& Ansari, D. (2014). Numerical predictors 
of arithmetic success in grades 1-6. Developmental Science, 17(5), 714-726. https://doi.org/10.1111/desc. 12152

Meissner, T. W., Walbrin, J., Nordt, M., Koldewyn, K., \& Weigelt, S. (2020). Head motion during fMRI tasks is reduced in children and adults if participants take breaks. Developmental Cognitive Neuroscience, 44, 100803. https://doi.org/10.1016/j.den.2020.100803

Nemmi, F., Schel, M. A., \& Klingberg, T. (2018). Connectivity of the Human Number Form Area Reveals Development of a Cortical Network for Mathematics. Frontiers in Human Neuroscience, 12(November), 1-15. https://doi.org/10.3389/fnhum.2018.00465

Park, J., Li, R., \& Brannon, E. M. (2014). Neural connectivity patterns underlying symbolic number processing indicate mathematical achievement in children. Developmental Science, 17(2), 187-202. https://doi.org/10.1111/desc.12114

Park, J., Park, D. C., \& Polk, T. A. (2013). Parietal functional connectivity in numerical cognition. Cerebral Cortex, 23(9), 2127-2135. https://doi.org/10.1093/cercor/bhs193

Price, G. R., \& Fuchs, L. S. (2016). The mediating relation between symbolic and nonsymbolic foundations of math competence. PLoS ONE, 11(2), 1-14. https://doi.org/10.1371/journal.pone.0148981

Price, G. R., Yeo, D. J., Wilkey, E. D., \& Cutting, L. E. (2018). Prospective relations between resting-state connectivity of parietal subdivisions and arithmetic competence. Developmental Cognitive Neuroscience, 30, 280-290. https://doi.org/10.1016/j.den.2017.02.006

Rosenberg-Lee, M., Ashkenazi, S., Chen, T., Young, C. B., Geary, D. C., \& Menon, V. (2015). Brain hyper-connectivity and operation-specific deficits during arithmetic problem solving 
in children with developmental dyscalculia. Developmental Science, 18(3), 351-372. https://doi.org/10.1111/desc. 12216

Rosenberg-Lee, M., Barth, M., \& Menon, V. (2011). What difference does a year of schooling make?. Maturation of brain response and connectivity between 2 nd and 3rd grades during arithmetic problem solving. NeuroImage, 57(3), 796-808.

https://doi.org/10.1016/j.neuroimage.2011.05.013

Rosenberg, M. D., Casey, B. J., \& Holmes, A. J. (2018). Prediction complements explanation in understanding the developing brain. Nature Communications, 9(1), 1-13. https://doi.org/10.1038/s41467-018-02887-9

Rosenberg, M. D., Finn, E. S., Scheinost, D., Papademetris, X., Shen, X., Constable, R. T., \& Chun, M. M. (2015). A neuromarker of sustained attention from whole-brain functional connectivity. Nature Neuroscience, 19(1), 165-171. https://doi.org/10.1038/nn.4179

Satterthwaite, T. D., Ciric, R., Roalf, D. R., Davatzikos, C., Bassett, D. S., \& Wolf, D. H. (2019). Motion artifact in studies of functional connectivity: Characteristics and mitigation strategies. Human Brain Mapping, 40(7), 2033-2051. https://doi.org/10.1002/hbm.23665

Ségonne, F., Pacheco, J., \& Fischl, B. (2007). Geometrically accurate topology-correction of cortical surfaces using nonseparating loops. IEEE Transactions on Medical Imaging, 26(4), 518-529. https://doi.org/10.1109/TMI.2006.887364

Shen, X., Finn, E. S., Scheinost, D., Rosenberg, M. D., Chun, M. M., Papademetris, X., \& Constable, R. T. (2017). Using connectome-based predictive modeling to predict individual behavior from brain connectivity. Nature Protocols, 12(3), 506-518. https://doi.org/10.1038/nprot.2016.178

Tung, K. C., Uh, J., Mao, D., Xu, F., Xiao, G., \& Lu, H. (2013). Alterations in resting functional 
connectivity due to recent motor task. NeuroImage, 78, 316-324.

https://doi.org/10.1016/j.neuroimage.2013.04.006

Whitfield-Gabrieli, S., \& Nieto-Castanon, A. (2012). Conn: A Functional Connectivity Toolbox for Correlated and Anticorrelated Brain Networks. Brain Connectivity, 2(3), 125-141. https://doi.org/10.1089/brain.2012.0073

Wilkey, E. D., \& Price, G. R. (2019). Attention to number: The convergence of numerical magnitude processing, attention, and mathematics in the inferior frontal gyrus. Human Brain Mapping, 40(3), 928-943. https://doi.org/10.1002/hbm.24422

Woodcock, R. W., McGrew, K. S., \& Mather, N. (2001). Woodcock-Johnson III Test. Riverside Publishing.

Yeo, B. T., Krienen, F. M., Sepulcre, J., Sabuncu, M. R., Lashkari, D., Hollinshead, M., Roffman, J. L., Smoller, J. W., Zöllei, L., Polimeni, J. R., Fisch, B., Liu, H., \& Buckner, R. L. (2011). The organization of the human cerebral cortex estimated by intrinsic functional connectivity. Journal of Neurophysiology, 106(3), 1125-1165. https://doi.org/10.1152/jn.00338.2011

Yeo, D. J., Wilkey, E. D., \& Price, G. R. (2017). The search for the number form area: A functional neuroimaging meta-analysis. Neuroscience and Biobehavioral Reviews, 78(January), 145-160. https://doi.org/10.1016/j.neubiorev.2017.04.027

Zhang, H., Wee, C. Y., Poh, J. S., Wang, Q., Shek, L. P., Chong, Y. S., Fortier, M. V., Meaney, M. J., Broekman, B. F. P., \& Qiu, A. (2019). Fronto-parietal numerical networks in relation with early numeracy in young children. Brain Structure and Function, 224(1), 263-275. https://doi.org/10.1007/s00429-018-1774-2

Zhao, H., Li, X., Karolis, V., Feng, Y., Niu, H., \& Butterworth, B. (2019). Arithmetic learning 
modifies the functional connectivity of the fronto-parietal network. Cortex, 111, 51-62. https://doi.org/10.1016/j.cortex.2018.07.016 
Table 1. Correlations among behavioral measures and in-scanner motion

\begin{tabular}{|c|c|c|c|c|c|c|c|c|c|c|}
\hline Variable $(N=31)$ & $M$ & $S D$ & Min & $\operatorname{Max}$ & 2 & 3. & 4. & 5 . & 6. & 7. \\
\hline 1.Age & 9.21 & 0.65 & 8.24 & 10.67 & & & & & & \\
\hline 2. Math Skills & 103.98 & 13.07 & 78.33 & $130.33-.234$ & & & & & & \\
\hline 3. Reading Skills & 107.97 & 11.36 & 82.50 & $127.50-.010$ & $.617 * * *$ & & & & & \\
\hline 4.FSIQ & 114.32 & 15.65 & 73.00 & $140.00-.089$ & $.56^{* *}$ & $.838 * * *$ & & & & \\
\hline 5. Symbolic Performance (P) & 1124.14 & 179.48 & 792.71 & $1555.31-.351$ & -.281 & $-.462 * *$ & $-.387^{*}$ & & & \\
\hline 6. Non-symbolic Performance (P) & 1167.58 & 216.87 & 760.90 & $1649.19-.127$ & $7-.229$ & $-.423 *$ & $-.385^{*}$ & $.465 * *$ & & \\
\hline 7. Symbolic Mean FD & 0.33 & 0.28 & 0.09 & $1.25-.051$ & -.257 & $-.456^{*}$ & -.335 & -.061 & .157 & \\
\hline 8. Non-Symbolic Mean FD & 0.27 & 0.20 & 0.09 & $0.82-.271$ & -.165 & $-.496 * *$ & -.209 & .201 & $.058 .407^{*}$ & \\
\hline 9. Rest Mean FD & 0.33 & 0.27 & 0.08 & $1.07-.039$ & -.274 & $-.451 *$ & -.200 & .056 & $.096 .749 * * *$ & $* .484 * *$ \\
\hline
\end{tabular}

${ }^{*} p<.05 ;{ }^{* *} p<.01 ; * * * p<.001$ 
Figure Captions

Figure 1. Connectome-based predictive models fits for each task. Scatter plots show the correlation between observed math skills scores outside the scanner and the math skills score predicted from functional connectivity during a given task. For each task separately, models were trained on connectivity data from $n-1$ children and tested on the held-out child, $n$. Note that the CPM includes both positive and negative connections as separate terms in the GLM. Each model controls for age, IQ, and mean FD. $* * p<.01$.

Figure 2. Connectome-based predictive model predicting math skills from functional connectivity during symbolic number comparison task. The left panel depicts connections with strengths that were positively correlated with children's math skills. The right panel depicts connections with strengths that were negatively correlated with children's math skills. Matrices represent the number of predictive connections within and between each canonical network as defined by Yeo et al., 2011. We display only consistent connections that correlated with math skills across all rounds of leave-one-out cross validation. Note that the CPM includes both positive and negative connections as separate terms in the GLM. Each model controls for age and mean FD.

Figure 3. Connectome-based predictive model predicting math skills from functional connectivity during resting-state. The left panel depicts connections with strengths that were positively correlated with children's math skills. The right panel depicts connections with strengths that were negatively correlated with children's math skills. Matrices represent the number of predictive connections within and between each canonical network as defined by Yeo 
et al., 2011. We display only consistent connections that correlated with math skills across all rounds of leave-one-out cross validation. Note that the CPM includes both positive and negative connections as separate terms in the GLM. Each model controls for age and mean FD. 